\title{
Matéria
}

ISSN 1517-7076

Revista Matéria, v. 11, n. 3, pp. 332 - 339, 2006

http://www.materia.coppe.ufrj.br/sarra/artigos/artigo10833

\section{Análise Térmica do Laminado PEEK/Carbono Submetido a Diferentes Rotas de Processamento}

\author{
M. C. A. Gatti ${ }^{1}$, R. V. da Silva ${ }^{2}$, J. R. Tarpani ${ }^{1}$ \\ 1 Escola de Engenharia de São Carlos-EESC-USP, Depto. de Enga de Materiais, Aeronáutica e \\ Automobilística, Av. Trabalhador São Carlense, 400, CEP:13566-590, São Carlos, SP, Brasil. \\ e-mail: mcgatti@sc.usp.br, jirpan@sc.usp.br \\ 2 Universidade Federal do Rio Grande do Norte, Centro de Tecnologia, Depto. de Enga Mecânica, Campus \\ Universitário, Lagoa Nova, CEP: 59072-970, Natal, RN, Brasil. \\ e-mail: rosanavilarim@yahoo.com.br
}

\section{RESUMO}

Laminados compostos de PEEK (poli-éter-éter-cetona) reforçados com fibras de carbono foram extraídos de placas de laminados híbridos metal-fibra (LMF) projetados para uso estrutural em aeronaves de alto desempenho, e re-processados segundo três rotas térmicas distintas daquela originalmente estabelecida. Os quatro laminados compostos resultantes de matriz termoplástica foram então extensivamente caracterizados por uma série de técnicas de análise térmica e termo-mecânica. Concluiu-se que a rota original de processamento do LMF gerou o laminado composto PEEK/C exibindo máximo desempenho mecânico em termos de módulo de elasticidade, bem como apresentando excelente estabilidade térmica/estrutural dentro do intervalo de temperatura previsto para o material em condições reais de operação em serviço.

Palavras chaves: Análise térmica, Material aeronáutico, Poli-éter-éter-cetona/PEEK, Polímero reforçado com fibras.

\section{Thermal Analysis of PEEK/Carbon Laminates Submitted to Different Processing Routes}

\section{ABSTRACT}

Carbon fiber reinforcing thermoplastic-PEEK composite laminates were extracted from fiber-metal laminates (FML) designed to structural applications in high performance aircrafts, and re-processed according three different thermal routes than that originally established. The four resulting PEEK/C laminates were then extensively characterized by thermal and thermo-mechanical techniques. It has been concluded that the original processing route of the FML gave rise to the PEEK/C composite exhibiting maximum mechanical performance in terms of elastic modulus, as well as presenting outstanding thermal/structural stability within the temperature range predicted for the material under in-service conditions.

Keywords: Aeronautical material, Fiber-reinforced thermoplastic polymer, PEEK/C composite, Thermal analysis.

\section{INTRODUÇÃO}

A Companhia Aeroespacial Boeing concebeu o laminado híbrido metal-fibra (LMF) TiGra composto por lâminas de titânio (Ti) e matriz polimérica termoplástica PEEK reforçada com fibras de carbono (ou Grafite), de modo a satisfazer as exigências estabelecidas para aplicações aeronáuticas em temperaturas relativamente elevadas, da ordem de $180^{\circ} \mathrm{C}$, tal como esperadas em fuselagens de aeronaves operando a Mach 2,5 (velocidade aproximada 2,5 vezes a do som), quando as ligas de alumínio de alta resistência não exibem desempenho mecânico aceitável [1]. As vantagens do TiGra derivam das combinações químicas, físicas, térmicas e mecânicas adequadas dos seus materiais constituintes. Os LMFs com polímeros termoplásticos reforçados aliam a resistência mecânica e à fadiga das fibras de carbono à ductilidade e tenacidade ao impacto do metal e à resistência e tolerância a danos e à corrosão do polímero, conduzindo a elevadas propriedades específicas (relativas à densidade) do material composto (ou compósito), 
e conseqüentemente a um alto grau de eficiência estrutural, características estas altamente desejáveis na indústria aeronáutica moderna.

Os polímeros termoplásticos de interesse para tais aplicações estruturais na indústria aeronáutica apresentam altas temperaturas de serviço. Dentre eles se destaca o PEEK (poli-éter-éter-cetona), com um potencial de uso contínuo até cerca de $250^{\circ} \mathrm{C}$, além de baixa emissão de fumaça e manutenção de resistência e tolerância a danos em temperaturas elevadas. O PEEK é um polímero aromático, semicristalino, com sua estrutura e propriedades finais, particularmente as mecânicas tais como resistências quase-estática, ao impacto (dinâmica) e à fadiga (cíclica), fortemente dependentes do processo de fabricação, em especial do ciclo térmico (taxa de resfriamento) aplicado, o qual determina em grande extensão o seu grau de cristalinidade, o tamanho das esferulitas e a morfologia e grau de perfeição dos cristalitos []].

Os LFMs utilizando polímeros termoplásticos podem ser conformados por compressão a quente, através da superposição de lâminas do metal e do composto pré-impregnado, seguida da prensagem a relativamente elevadas temperaturas e pressões para fusão do polímero, e resfriamento controlado para consolidação do produto final nos padrões almejados.

Conclui-se, portanto, que as propriedades térmicas e mecânicas finais destes laminados híbridos estruturais dependem em grande extensão do ciclo térmico empregado no seu processo de fabricação.

Visto que as características e propriedades finais da fase polimérica reforçada com as fibras de carbono são extremamente susceptíveis ao ciclo térmico empregado na fabricação dos LMFs, torna-se importante avaliarem-se suas propriedades térmicas e termo-reológicas considerando-se as diferentes rotas de tratamento térmico impostas durante a fabricação do laminado metal-fibra. As chamadas técnicas de análise térmica mostram-se bastante úteis para este fim.

Análise térmica é uma descrição geral para um grupo de técnicas nas quais alguma propriedade física do material é continuamente registrada enquanto o mesmo é aquecido (ou resfriado), em geral sob uma taxa constante. As técnicas de análise térmica mais comumente empregadas na caracterização de polímeros, geralmente puros, ou seja, não carregados ou reforçados, são a Termogravimetria (TGA), a Calorimetria Exploratória Diferencial (DSC) e a Análise Térmica Dinâmico Mecânica (DMTA). Uma descrição detalhada destas técnicas e de seus potenciais aplicativos é fornecida em [ㄱ]

Objetiva-se neste estudo analisar o comportamento térmico e termo-mecânico do polímero PEEK (poli-éter-éter-cetona), reforçado com fibras de carbono, através das técnicas de Análise Termogravimétrica (TGA), Calorimetria Exploratória Diferencial de Varredura (DSC) e Análise Térmica Dinâmico-Mecânica (DMTA), para diferentes condições de processamento do material.

\section{MATERIAL E TRATAMENTOS TÉRMICOS}

Em meados do ano de 2003, placas do laminado TiGra foram obtidas, em nível de laboratório, intercalando-se lâminas de titânio comercialmente puro com camadas de fibras unidirecionais de grafite préimpregnadas com PEEK, sendo a consolidação final por compressão a quente em molde fechado, seguida por resfriamento controlado. O ciclo de processamento do material foi elaborado e desenvolvido na Universidade de Liverpool, estando atualmente sob sigilo comercial.

Pequenos tabletes com dimensões no plano de 10 x $55 \mathrm{~mm}^{2}$ e espessura integral $(\sim 4,8 \mathrm{~mm})$ foram extraídos das placas originais de TiGra através de corte com jato d’água, e submetidos a um novo e idêntico ciclo de compressão mecânica a quente tal como originalmente estabelecido para o LMF (i.e., mesmas taxas de aquecimento, temperatura máxima alcançada e pressão e tempo de compressão), porém com a subseqüente aplicação de três distintas rotas alternativas de resfriamento, bastante dissimilares àquela original, quais sejam, respectivamente: recozimento ao forno, resfriamento brusco em água, e resfriamento brusco em nitrogênio líquido.

De posse dos tabletes nas quatro diferentes condições térmicas ( 1 original e 3 alternativas), foram deles cuidadosamente retirados os laminados compostos PEEK/C (correspondentes à porção em destaque na Figura 1) por intermédio de estiletes afiados e bastante resistentes. Os laminados compostos de matriz termoplástica reforçados com fibras cerâmicas, com espessura 1,1 mm e dimensões no plano de 10 x 55 $\mathrm{mm}^{2}$, eram constituídos por sete camadas ou lâminas sucessivas e consolidadas de pré-impregnado com fibras de reforço unidirecionais, seguindo o arranjo $\left[(0)_{7}\right]_{\mathrm{T}}$, fornecido pela ICI (nominalmente com $60 \%$ em volume de fibras).

Destes laminados PEEK/C foram então finalmente obtidos os corpos de prova e as amostras descritos no item 3 e utilizados no presente trabalho. 


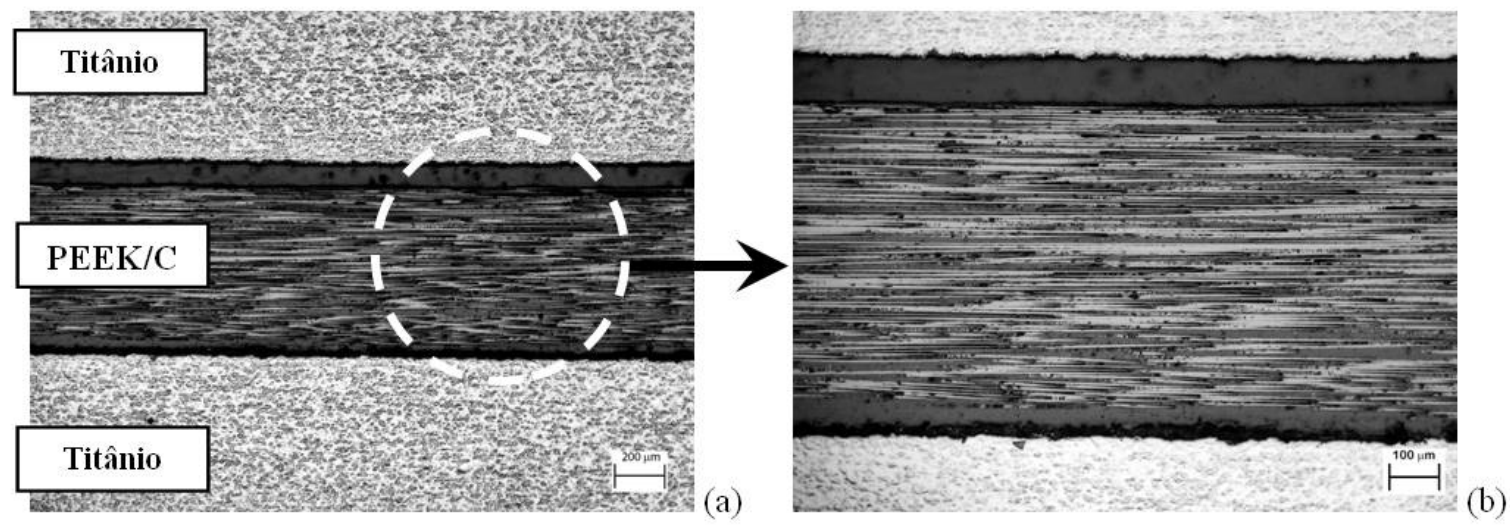

Figura 1: Microconstituintes típicos do laminado TiGra (a), e do composto PEEK/C (b).

\section{MÉTODOS}

\subsection{Análise Termogravimétrica (TGA)}

As análises foram realizadas num aparelho DuPont Instruments 951 Thermogravimetric Analysis, no intervalo de temperaturas de 0 a $900^{\circ} \mathrm{C}$, sob uma taxa de aquecimento de $10^{\circ} \mathrm{C} / \mathrm{min}$ e fluxo constante de nitrogênio $(100 \mathrm{ml} / \mathrm{min}$ ), dispondo-se a amostra (com aproximadamente $8 \mathrm{mg}$ ) em panela de platina. A análise foi realizada em triplicata, e refere-se somente a amostras na condição de como-fabricado.

\subsection{Calorimetria Exploratória Diferencial de Varredura (DSC)}

As análises foram feitas num aparelho DuPont Instruments 910 Differential Scanning Calorimeter, no intervalo de 0 a $400^{\circ} \mathrm{C}$, sob uma taxa de aquecimento de $5^{\circ} \mathrm{C} / \mathrm{min}$ e fluxo constante de nitrogênio (100 $\mathrm{ml} / \mathrm{min}$ ), utilizando-se amostras ( $7 \mathrm{mg}$ ) mantidas em panela de alumínio hermeticamente fechada. Foram realizadas quatro análises para cada uma das quatro distintas condições térmicas (como-fabricado + três rotas alternativas de resfriamento) em que se encontrava o composto PEEK/C.

\subsection{Análise Térmica Dinâmico-Mecânica (DMTA)}

As análises foram conduzidas num aparelho DuPont Instruments 983 Dynamic Mechanical Analysis, entre 0 e $250^{\circ} \mathrm{C}$, sob uma taxa de aquecimento de $5^{\circ} \mathrm{C} / \mathrm{min}$. O carregamento mecânico foi do tipo barra duplamente engastada, com um movimento oscilatório relativo entre os pontos de engaste de $0,5 \mathrm{~mm}$ imposto na forma de onda senoidal a uma freqüência fixa de $1 \mathrm{~Hz}$.

Utilizaram-se espécimes com dimensões aproximadas de $1,1 \times 10 \times 55 \mathrm{~mm}^{3}$, sendo o vão entre os pontos de aplicação de carga de $25 \mathrm{~mm}$. Foram realizados dois ensaios para cada uma das quatro condições térmicas avaliadas do laminado PEEK/C.

\section{RESULTADOS E DISCUSSÃO}

\subsection{Análise Termogravimétrica}

A Figura 2 apresenta os resultados obtidos durante os experimentos de termogravimetria do material no estado como-fabricado.

Observa-se que o composto PEEK/C é termicamente estável até a temperatura de $560^{\circ} \mathrm{C}$ sem que ocorra perda detectável de massa durante o aquecimento. Após os $560^{\circ} \mathrm{C}$ inicia-se o processo de decomposição térmica do polímero e, eventualmente, dos seus agentes de acoplagem às fibras de reforço, ocorrendo perda de massa da ordem de $16 \%$ entre 560 e $675^{\circ} \mathrm{C}$. A partir deste ponto, observa-se uma perda de massa contínua, porém menos acelerada, do material orgânico até os $900^{\circ} \mathrm{C}$. A estabilidade térmica da fibra de carbono em atmosfera inerte de $\mathrm{N}_{2}$ é extremamente alta se comparado ao PEEK, não obstante sua degradação térmica em meio oxidante possa iniciar-se já a partir dos $600^{\circ} \mathrm{C}$ [4]. 
Conclui-se assim que, termicamente, o material em estudo suporta temperaturas de até $560^{\circ} \mathrm{C}$ sem sofrer qualquer instabilidade que leve à perda de massa.

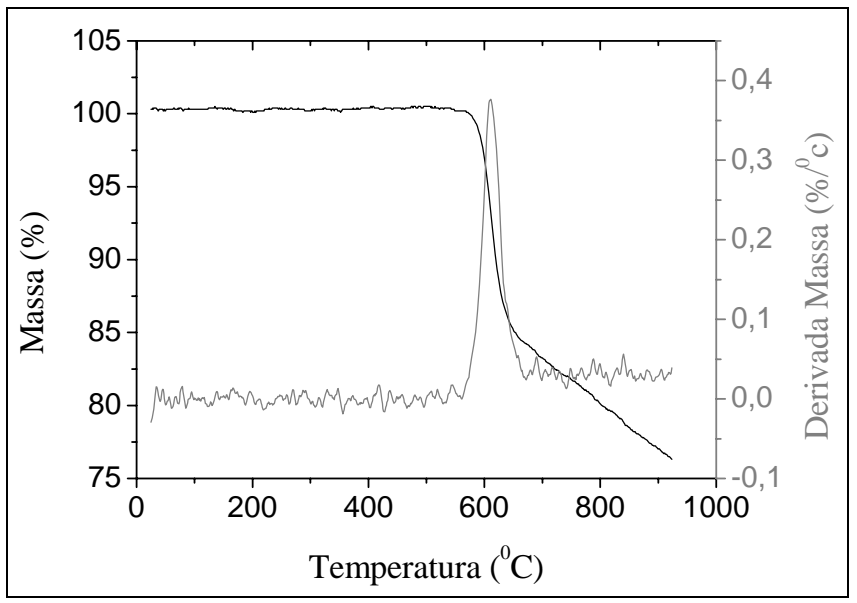

Figura 2: Curva termogravimétrica do PEEK/carbono original.

\subsection{Calorimetria Exploratória Diferencial de Varredura}

A Figura 3 apresenta as curvas relativas ao fluxo de calor versus temperatura, levantada durante os ensaios de DSC. A Tabela 1 lista os resultados obtidos para o composto PEEK/C nas diferentes condições de tratamento térmico.

Como esperado, a temperatura de transição vítrea dos compostos é superior à do PEEK puro devido à presença das fibras de carbono, que restringem a livre movimentação das macromoléculas. Além disso, a superfície da fibra de carbono atua como agente de nucleação, favorecendo a cristalização local do PEEK [5, 6] e, portanto, contribuindo para o aumento da $\mathrm{T}_{\mathrm{G}}$.

Avaliando-se o ranqueamento dos valores de $\mathrm{T}_{\mathrm{G}}$, nota-se uma tendência de seu aumento com a diminuição da taxa de resfriamento aplicada no tratamento térmico do composto, o que é condizente com a literatura consultada [6]. Resfriamentos mais lentos reconhecidamente favorecem a cristalização dos polímeros, aumentando assim sua $\mathrm{T}_{\mathrm{G}}$. Um comportamento algo contraditório parece, em princípio, ocorrer relativamente aos materiais resfriados em água e nitrogênio; porém, como a ser observado mais tarde no texto, água se mostrou um meio refrigerante mais eficiente frente ao $\mathrm{N}_{2}$ líquido.

Em temperaturas acima de $T_{G}$ as cadeias do PEEK têm mobilidade suficiente para se reorganizarem, havendo a possibilidade da formação das regiões cristalinas durante o aquecimento lento da amostra durante o ensaio, com o aparecimento de um correspondente pico exotérmico relativo à temperatura de cristalização $\left(\mathrm{T}_{\mathrm{C}}\right)$. Como mostra a Figura 3, isto só não ocorreu na amostra recozida, visto ela ter praticamente exaurido seu potencial de cristalização no ciclo de resfriamento que precedeu a análise de DSC. A acentuada diferença dos valores obtidos de entalpia de cristalização $\left(\Delta \mathrm{H}_{\mathrm{C}}\right)$ está provavelmente relacionada às distintas taxas de resfriamento empregadas durante os vários tratamentos térmicos aplicados ao PEEK/C.

Acima de $\mathrm{T}_{\mathrm{C}}$, os cristais recentemente formados e os remanescentes do ciclo de resfriamento de fabricação sofrem fusão, e um pico endotérmico, relativo à temperatura de fusão cristalina $\left(\mathrm{T}_{\mathrm{M}}\right)$ é observado. Das curvas mostradas na Figura 3, a entalpia de fusão apresenta valores relativamente próximos entre si, da ordem dos $18 \mathrm{~J} / \mathrm{g}$. Em todas as análises, $\mathrm{T}_{\mathrm{M}}$ apresentou valores muito próximos, indicando certa independência desta variável com o tratamento térmico previamente aplicado. No entanto, o mínimo valor de $\mathrm{T}_{\mathrm{M}}$ exibido pelo material recozido, assim como o máximo de $\mathrm{T}_{\mathrm{M}}$ referente àquele resfriado em água, são condizentes com a literatura [7], no sentido de que esferulitas e cristalitos com maior grau de perfeição, gerados sob resfriamentos mais lentos, tendem a fundir sob temperaturas mais baixas, e vice-e-versa.

Como já referido, é amplamente aceito que o grau de cristalinidade de um dado polímero depende da sua taxa de resfriamento durante o processo de solidificação. Taxas mais lentas favorecem o movimento e alinhamento das macro-moléculas, e o conseqüente arranjo mais elaborado e regular da estrutura final. Como esperado, a amostra que apresentou maior grau de cristalinidade foi a recozida, próximo de $37 \%$ em massa (ou $\sim 35 \%$ em volume). 


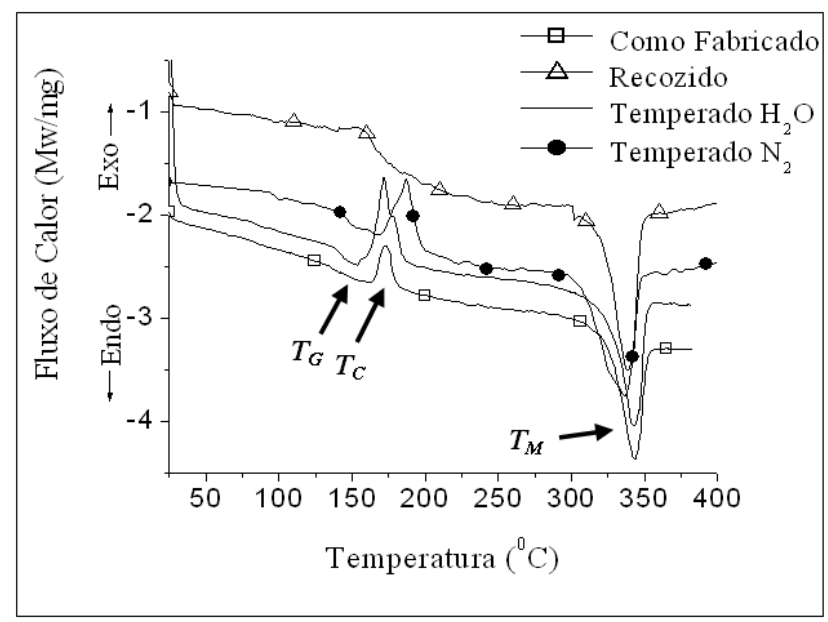

Figura 3: Curvas de DSC das amostras de PEEK/C submetidas a distintos tratamentos térmicos em rotas original e alternativas de fabricação.

Tabela 1: Temperaturas de transição vítrea $\left(T_{G}\right)$, de cristalização exotérmica $\left(T_{C}\right)$ e de fusão cristalina endotérmica $\left(\mathrm{T}_{\mathrm{M}}\right)$, entalpias de cristalização $\left(\Delta \mathrm{H}_{\mathrm{C}}\right)$ e de fusão cristalina $\left(\Delta \mathrm{H}_{\mathrm{M}}\right)$, e porcentual estimado de cristalinidade nas diferentes amostras avaliadas por DSC (valores médios).

\begin{tabular}{|c|c|c|c|c|c|c|}
\hline Amostra & $\begin{array}{c}\mathrm{T}_{\mathrm{G}} \\
\left({ }^{\circ} \mathrm{C}\right)\end{array}$ & $\begin{array}{c}\mathrm{T}_{\mathrm{C}} \\
\left({ }^{\circ} \mathrm{C}\right)\end{array}$ & $\begin{array}{c}\mathrm{T}_{\mathrm{M}} \\
\left({ }^{\circ} \mathrm{C}\right)\end{array}$ & $\begin{array}{c}\Delta \mathrm{H}_{\mathrm{C}} \\
(\mathrm{J} / \mathrm{g})\end{array}$ & $\begin{array}{c}\Delta \mathrm{H}_{\mathrm{M}} \\
(\mathrm{J} / \mathrm{g})\end{array}$ & $\begin{array}{c}\% \text { massa de } \\
\text { cristalinidade }^{\mathrm{a}}\end{array}$ \\
\hline PEEK puro [8] & 144 & 169 & 339 & ---- & ---- & $\sim 48$ (máx.) \\
\hline Como-fabricada & 160 & 173 & 343 & 2,9 & 19,9 & 33 \\
\hline Recozida & 163 & ---- & 336 & 0,1 & 19,5 & 37 \\
\hline Temperada em $\mathrm{H}_{2} \mathrm{O}$ & 150 & 171 & 347 & 8,0 & 17,4 & 16 \\
\hline Temperada em $\mathrm{N}_{2}$ & 153 & 180 & 338 & 9,3 & 15,4 & 16 \\
\hline
\end{tabular}

${ }^{a}$ Estimado com base na diferença entre os picos exo- e endotérmicos mostrados na Fig. 3. A ser confirmado por microscopia óptica de transmissão de luz polarizada.

\subsection{Análise Térmica Dinâmico-Mecânica}

As curvas de $\tan \delta$ e do módulo de armazenamento (E’) em função da temperatura, determinadas nos ensaios de DMTA, são apresentadas nas Figuras 4 e 5, respectivamente. Os valores da temperatura de transição vítrea $\left(\mathrm{T}_{\mathrm{G}}\right)$ determinada no ponto de máximo de tan $\delta$, e dos módulos de armazenamento (E') e de perda (E”), estes derivados na temperatura de $100^{\circ} \mathrm{C}$ (pertencente ao intervalo de aplicação em serviço dos LMFs), são listados na Tabela 2. Merece ser destacado que o valor da $\tan \delta$ (= E’/E') é uma medida do grau de amortecimento mecânico do material, e, quanto mais dúctil ou tenaz o material, maior o seu grau de amortecimento. Materiais com alto amortecimento dissipam grande parte da energia que foi utilizada no seu

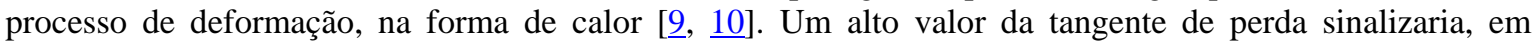
princípio, para um material polimérico propenso à redução de suas propriedades mecânicas em serviço (baixas estabilidades estrutural e, eventualmente, dimensional) como decorrência da forte dissipação de calor. Isto seria indesejável, por exemplo, em estruturas que suportam cargas por longos períodos de tempo. Sob esta óptica, no caso em questão, a amostra mais indesejável seria, portanto, a temperada em $\mathrm{H}_{2} \mathrm{O}(\tan \delta=$ 0,205). Também neste sentido, a amostra que apresentou a tangente de perda mais atrativa é a recozida, seguida muito proximamente da como-fabricada. 


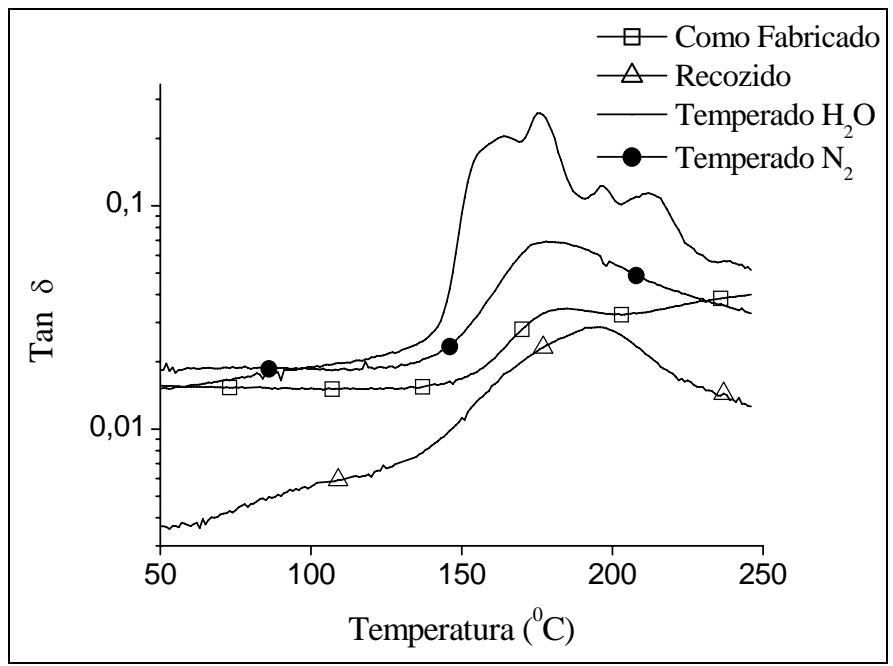

Figura 4: Variação de $\tan \delta$ com a temperatura do ensaio.

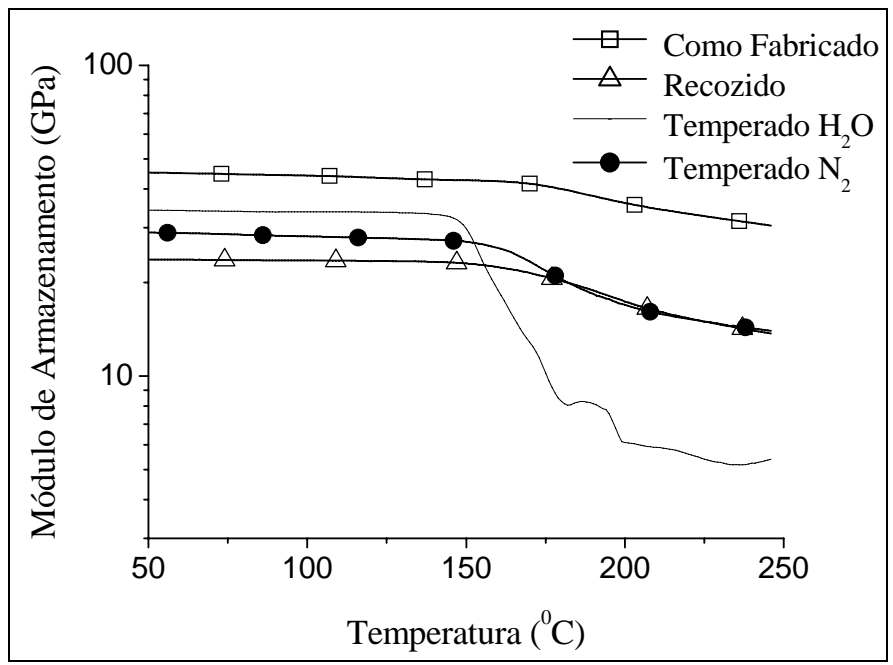

Figura 5: Variação do módulo de armazenamento, E’, com a temperatura de ensaio.

Tabela 2: Temperatura de transição vítrea $\left(\mathrm{T}_{\mathrm{G}}\right)$, estimada através do pico de tan $\delta$, e módulos de armazenamento (E') e de perda (E'”) à $100^{\circ} \mathrm{C}$ para as diferentes condições de tratamento térmico (valores médios).

\begin{tabular}{|c|c|c|c|c|}
\hline Amostra & $\begin{array}{c}\mathrm{T}_{\mathrm{G}}\left({ }^{\circ} \mathrm{C}\right) \\
\text { (máximo de } \tan \delta)\end{array}$ & $\begin{array}{c}\text { Pico de } \\
\tan \delta\end{array}$ & E' (GPa) & $\mathrm{E}^{\prime}(\mathrm{MPa})^{\mathrm{b}}$ \\
\hline Como-fabricada & 185 & 0,035 & 44,2 & 672 \\
\hline Recozida & 190 & 0,037 & 23.6 & 640 \\
\hline Temperada em $\mathrm{H}_{2} \mathrm{O}$ & 176 & 0,260 & 33,7 & 377 \\
\hline
\end{tabular}

${ }^{b}$ Valores possivelmente superestimados como decorrência da geometria de carregamento adotada, muito restritiva à deformação do espécime se comparada, por exemplo, à flexão em três pontos, especialmente quando considerados a grande diferença entre os coeficientes de expansão térmica entre o material ensaiado e o que constitui os suportes, e o amplo intervalo de temperatura amostrado nos testes [11]. 
Como esperado [12], os valores da temperatura de transição vítrea determinados por DMTA são bem superiores aos obtidos via DSC, porém as seqüências de valores determinadas por ambas as técnicas são idênticas. Novamente, a maior $\mathrm{T}_{\mathrm{G}}$ é do composto recozido e a menor pertence ao temperado em água. Estes resultados corroboram a expectativa, com base na literatura, de que os materiais solidificados mais lentamente a partir do estado fundido, por gerarem áreas cristalinas mais extensas, restringem mais efetivamente a mobilidade das macromoléculas nas zonas amorfas, e, por conseguinte, maiores temperaturas de transição vítrea são estabelecidas [1].

Com relação ao módulo de armazenamento, também denominado módulo elástico, observa-se uma forte redução para a amostra temperada em $\mathrm{H}_{2} \mathrm{O}$ a partir de $150^{\circ} \mathrm{C}$, já na região de transição vítrea. Este comportamento está certamente relacionado ao processo de alívio das tensões residuais internas, congeladas durante a etapa de resfriamento brusco do laminado composto e principais responsáveis pelo relativamente elevado valor de E' alcançado por intermédio deste tratamento térmico, comparativamente aos laminados resfriados em nitrogênio e ao forno (recozido), respectivamente.

Vale citar que a técnica de DMTA é, de fato, uma poderosa ferramenta na análise do grau de tensões internas congeladas durante o processamento de peças poliméricas. Através de ensaios amostrando uma ampla faixa de temperaturas, é possível inferir o processo de relaxação das tensões internas da peça por intermédio da queda acentuada do seu módulo de armazenamento, além de um expressivo aumento do amortecimento (pico de tan $\delta$ ), se comparados valores obtidos a partir de uma amostra não tensionada [14], exatamente como verificado nos experimentos ora apresentados.

Nota-se na Figura 5 que, nos compostos recozido e como-fabricado (em especial neste último), a redução de E' na região de transição vítrea é muito menos comprometedora que nos laminados resfriados bruscamente (particularmente naquele resfriado em água), em ambos os níveis percentual e absoluto, denotando assim a boa estabilidade estrutural dos primeiros materiais num intervalo vital de temperaturas previsto para os LMFs em serviço. Neste contexto, vale destacar que o módulo elástico do laminado comofabricado se mantém muitíssimo superior (note a escala logarítmica) a todos os demais, em especial nas proximidades da temperatura de $180^{\circ} \mathrm{C}$, que é a máxima estimada para o uso contínuo e estrutural a que os laminados híbridos se destinam em serviço. Este fato confirma a otimização microestrutural do laminado como-fabricado, no sentido da maximização das propriedades termo-mecânicas presentemente avaliadas. Um dos fatores responsáveis por tal desempenho seria o alto grau de cristalinidade da matriz polimérica, o qual, em parte, certamente se desenvolveu a partir da superfície das fibras de reforço, favorecendo assim elevadas resistências de coesão interfacial fibra/matriz, colaborando, portanto, na capacidade do material resistir a cargas mecânicas. Além disso, há de se enfatizar que estruturas altamente ordenadas impõem naturalmente maiores restrições aos mecanismos de deformação, o que, neste caso, se refletiria diretamente na resistência mecânica e dureza da matriz polimérica. No entanto, há de se ressaltar a tendência observada na literatura [7] da redução do potencial global de cristalização do polímero reforçado, frente ao exibido pelo mesmo polímero puro, para condições idênticas de processamento, não obstante ainda a superfície das fibras atue como local preferencial de nucleação das esferulitas.

Permanece ainda por explicar o relativamente baixo desempenho exibido pelo material recozido frente ao como-fabricado, visto que, ao menos no tocante ao quesito cristalinidade, o primeiro atingiu o teor máximo dentre todos os avaliados. Fundamental para se responder tal questionamento, serão as análises microestruturais já previstas em microscópio óptico de transmissão.

Como última observação, vale mencionar que todos os resultados obtidos $\left(T_{G}, T_{M}\right.$, pico tan $\left.\delta, E^{\prime}\right)$ indicaram a água como um meio refrigerante mais efetivo que o nitrogênio líquido para o resfriamento do composto PEEK/carbono, ou seja, ela apresentou uma maior eficiência na extração de calor das amostras previamente aquecidas. Tal fato justificaria assim o procedimento comumente adotado para a têmpera subzero de produtos metalúrgicos, quando, anteriormente à imersão do componente ao rubro em nitrogênio líquido, o mesmo é previamente imerso em água.

\section{CONCLUSÃO}

Foi demonstrado neste estudo que a rota de processamento empregada na fabricação de laminados metal-fibra TiGra para uso aeronáutico gerou, com base nos resultados alcançados em análises térmicas e termo-mecânicas, uma microestrutura otimizada da fase composta de PEEK e fibras fortalecedoras de carbono. Neste sentido, as propriedades mecânicas do produto dito original, ou como-fabricado, se mostraram substancialmente superiores, e termicamente mais estáveis, quando comparadas àquelas obtidas através de rotas térmicas alternativas. 


\section{AGRADECIMENTOS}

À Capes pela bolsa de estudos de doutorado de M.C.A.G., e ao CNPq pela bolsa de estudos de desenvolvimento regional de R.V.S..Um dos autores, J.R.T., gostaria de expressar seu particular reconhecimento e apreciação pelas facilidades disponibilizadas pela Universidade de Liverpool-UK, em especial direcionados ao Prof. James Wesley Cantwell.

\section{BIBLIOGRAFIA}

[1] BURIANEK, D.A., "Laboratory for Advanced Composites", Department of Aeronautics and Astronautics, MIT, Cambridge, Private Document, 2002.

[2] MORALES, A.R., "PEI/PEEK/Liquid Crystalline Polymer Ternary Blends”, European Polymer Journal, v. 32, pp. 349-63, 1996.

[3] CANEVAROLO, S.V., Técnicas de Caracterização de Polímeros, Artliber Ed., v. 1, São Paulo, 2003.

[4] HALBIG, M.C., "Degradation of Continuous Fiber Ceramic Matrix Composites", Symp. Environmental, Mechanical, and Thermal Properties and Performance of Continuous Fiber Ceramic Composite, Seattle, WA, 1999.

[5] SEFERISI, J.C., "A Collaborative Study of the Structure and Morphology in PET and PEEK”, Pure \& Applied Chemical, v. 65, pp. 1581-93, 1993.

[6] GUNILLA, M.K., "Annealing Effects on the Crystallinity of PEEK and its Carbon Fiber Composite", Journal of Applied Polymer Science, v. 33, pp. 332-337, 1987.

[7] SHANG-LIN, G., "Cooling Rates Influences in Carbon Fibre/PEEK Composites - Part I”, Composites: Part A, v. 31, pp. 517-530, 2000.

[8] LI, R., "Time-temperature Superposition Method for Glass Transition Temperature of Plastic Materials", Materials Science Engineering A, v. 278, pp. 36-45, 2000.

[9] CANEVAROLO, S.V., "Análise Dinâmico-mecânica para Sistemas Poliméricos. Parte 1: O Amortecimento”, ABPol, Maio, 1991.

[10] HERTZBERG, R.W., Deformation and Fracture Mechanics of Engineering Materials, John Wiley \& Sons, Ed., New York, NY, pp. 206-210, 1976.

[11] MELO, J.D.D., "Time and Temperature Dependence of the Viscoelastic Properties of PEEK/IM7", Journal of Composite Materials, v. 38, pp. 1815-1830, 2004.

[12] HATAKEYAMA, T., Thermal Analysis, Chichester, John Wiley, 2002.

[13] VAUGHAN, A.S., "Irradiation and the Glass Transition in PEEK”, Polymer, v. 42, pp. 8891-8895, 2001.

[14] CANEVAROLO, S.V., “Avaliação do Grau de Tensão Interna Congelada Durante o Processamento de Peças Poliméricas Moldadas”, Boletim 6 ABPol, pp. 41-42, Maio, 1991. 